TRANSACTIONS OF THE

AMERICAN MATHEMATICAL SOCIETY

Volume 349, Number 6, June 1997, Pages 2493-2505

$\mathrm{S} 0002-9947(97) 01848-5$

\title{
THE FLOER HOMOTOPY TYPE OF HEIGHT FUNCTIONS ON COMPLEX GRASSMANN MANIFOLDS
}

\author{
DAVID E. HURTUBISE
}

\begin{abstract}
A family of Floer functions on the infinite dimensional complex Grassmann manifold is defined by taking direct limits of height functions on adjoint orbits of unitary groups. The Floer cohomology of a generic function in the family is computed using the Schubert calculus. The Floer homotopy type of this function is computed and the Floer cohomology which was computed algebraically is recovered from the Floer homotopy type. Certain non-generic elements of this family of Floer functions were shown to be related to the symplectic action functional on the universal cover of the loop space of a finite dimensional complex Grassmann manifold in the author's preprint The Floer homotopy type of complex Grassmann manifolds.
\end{abstract}

\section{INTRODUCTION}

Recently Cohen, Jones, and Segal have been trying to develop an underlying homotopy theory for Floer homology [6], [8]. According to their theory a Floer function, i.e. a function that can be used to define Floer homology groups [9], [10], [11], should in many cases also give rise to an inverse system of spectra (a prospectrum). They call this inverse system of spectra the "Floer homotopy type", and the Floer homology groups should be recoverable from the Floer homotopy type. The analogous situation in Morse theory is that a Morse function gives rise to a CW complex whose homology agrees with the Morse homology [14], [16].

In [8] Cohen, Jones, and Segal considered the example of a Floer function $f$ : $\mathbb{C} P^{\infty} \rightarrow \mathbb{R}$. This function is a modification, due to Segal, of the Morse function on $\mathbb{C} P^{k}$ defined in [14]. Segal's idea was to use the function which appears in [14] extended to $\mathbb{C} P^{\infty}$ but with the basis elements of $\mathbb{C}^{\infty}$ indexed over $\mathbb{Z}$ instead of $\mathbb{Z}^{+}$. The result is a function on $\mathbb{C} P^{\infty}$ whose critical points all have infinite index but whose relative indices are all finite. The Floer cohomology of $f: \mathbb{C} P^{\infty} \rightarrow \mathbb{R}$ has the structure of a module over $H^{*}\left(\mathbb{C} P^{\infty}\right)$. This Floer cohomology module is $H^{*}\left(\mathbb{C} P^{\infty}\right)\left[c_{1}^{-1}\right]$, where $c_{1}$ is the Chern class of the Hopf line bundle $\gamma_{1}$ over $\mathbb{C} P^{\infty}$. The Floer homotopy type of $\mathbb{C} P^{\infty} \rightarrow \mathbb{R}$ is

$$
\mathbb{C} P^{\infty} \leftarrow\left(\mathbb{C} P^{\infty}\right)^{-\gamma_{1}} \leftarrow\left(\mathbb{C} P^{\infty}\right)^{-2 \gamma_{1}} \leftarrow \cdots
$$

and the Floer cohomology module can be recovered from this pro-spectrum using the Thom Isomorphism Theorem as the direct limit of the following:

$$
H^{*}\left(\mathbb{C} P^{\infty}\right) \stackrel{\cup c_{1}}{\longrightarrow} H^{*+2}\left(\mathbb{C} P^{\infty}\right) \stackrel{\cup c_{1}}{\longrightarrow} H^{*+4}\left(\mathbb{C} P^{\infty}\right) \stackrel{\cup c_{1}}{\longrightarrow} \cdots .
$$

Received by the editors January 29, 1996.

1991 Mathematics Subject Classification. Primary: 55P15; Secondary 58B05, 58F09.

Research supported by an NSF graduate fellowship. 
In this paper I define a family of Floer functions on $G_{n}\left(\mathbb{C}^{\infty}\right)$, the infinite dimensional complex Grassmann manifold. These Floer functions are direct limits of well known height functions defined on certain adjoint orbits of $U_{n+k}$ diffeomorphic to $G_{n, n+k}(\mathbb{C})$ [4]. An explicit formula for the gradient flow lines of these height functions with respect to the Killing form is derived, and the functions are shown to satisfy the Morse-Bott-Smale transversality condition (see Theorems 3 and 5). For a generic height function on $G_{n, n+k}(\mathbb{C})$ I show that the unstable manifolds of the function are the Schubert cells of $G_{n, n+k}(\mathbb{C})$ (see Theorem 8).

One function $f_{A}: G_{n}\left(\mathbb{C}^{\infty}\right) \rightarrow \mathbb{R}$ in the family is an extension of the function studied by Cohen, Jones, and Segal in [8]. This function has critical points whose Morse indices are all infinite; however, the relative index between any two points is finite. Using the relative index (see Definition 10), the Floer chain complex of $f_{A}: G_{n}\left(\mathbb{C}^{\infty}\right) \rightarrow \mathbb{R}$ is defined. There is a natural filtration of the Floer chain complex such that the cohomology of each term in the filtration is isomorphic (after reindexing) to $H^{*}\left(G_{n}\left(\mathbb{C}^{\infty}\right)\right)$. This isomorphism induces a direct system of chain complexes, and the cohomology of the direct limit is computed as a module over $H^{*}\left(G_{n}\left(\mathbb{C}^{\infty}\right)\right)$ using the Schubert calculus. This cohomology module is the localization of $H^{*}\left(G_{n}\left(\mathbb{C}^{\infty}\right)\right)$ at $c_{n}$, the $n$th Chern class of the tautological $n$-plane bundle $\gamma_{n}$ over $G_{n}\left(\mathbb{C}^{\infty}\right)$, i.e.

$$
H F^{*}\left(G_{n}\left(\mathbb{C}^{\infty}\right)\right) \approx H^{*}\left(G_{n}\left(\mathbb{C}^{\infty}\right)\right)\left[c_{n}^{-1}\right] .
$$

Each term in the filtered Floer chain complex has the cohomology of $G_{n}\left(\mathbb{C}^{\infty}\right)^{-j \gamma_{n}}$ for some $j \in \mathbb{Z}_{+}$. Moreover, the maps in the direct system of chain complexes coming from the filtration are multiplication by the Euler class of $\gamma_{n}$. This algebraic information is reflected in the Floer homotopy type of $f_{A}$. The Floer homotopy type of $f_{A}: G_{n}\left(\mathbb{C}^{\infty}\right) \rightarrow \mathbb{R}$ has

$$
G_{n}\left(\mathbb{C}^{\infty}\right) \leftarrow G_{n}\left(\mathbb{C}^{\infty}\right)^{-\gamma_{n}} \leftarrow G_{n}\left(\mathbb{C}^{\infty}\right)^{-2 \gamma_{n}} \leftarrow \cdots
$$

as a cofinal system, where the maps are induced by certain bundle inclusions. The Floer cohomology module can be recovered from this pro-spectrum using the Thom Isomorphism Theorem as the direct limit of the following:

$$
H^{*}\left(G_{n}\left(\mathbb{C}^{\infty}\right)\right) \stackrel{\cup c_{n}}{\longrightarrow} H^{*+2 n}\left(G_{n}\left(\mathbb{C}^{\infty}\right)\right) \stackrel{\cup c_{n}}{\rightarrow} H^{*+4 n}\left(G_{n}\left(\mathbb{C}^{\infty}\right)\right) \stackrel{\cup c_{n}}{\longrightarrow} \cdots
$$

The Floer homotopy type of $f_{A}: G_{n}\left(\mathbb{C}^{\infty}\right) \rightarrow \mathbb{R}$ is constructed from its flow category [7], [13]. The flow category of $f_{A}$ is a topological category whose objects are the critical points of $f_{A}$ and whose morphisms are the unparameterized piecewise gradient flow lines of $f_{A}$. The flow category of $f_{A}: G_{n}\left(\mathbb{C}^{\infty}\right) \rightarrow \mathbb{R}$ is particularly easy to work with, in part because its morphism spaces are compact. The flow category associated to a certain non-generic element of the family of Floer functions defined in this paper is related to the flow category of the symplectic action functional on the universal cover of the loop space of $G_{n, n+k}(\mathbb{C})$ via a group action and an $\mathbb{R}$ equivariant fiber bundle. For the case $n=1$ the group action is trivial and the flow category of this non-generic function compactifies the flow category of the action functional on the universal cover of the loop space of $\mathbb{C} P^{\infty}$. Details concerning the relationship between the flow categories defined in this paper and the flow category of the symplectic action functional can be found in [13]. 


\section{HeIGHT FUNCTIONS ON ADJOINT ORBITS}

Let $G$ be a compact connected semi-simple Lie group with Lie algebra $\mathfrak{g}$. Let $x_{0} \in \mathfrak{g}$ and consider $G / G_{x_{0}} \approx G \cdot x_{0} \subseteq \mathfrak{g}$, where $\cdot$ denotes the adjoint action. For any $A \in \mathfrak{g}$ define $f_{A}: G \cdot x_{0} \rightarrow \mathbb{R}$ by the formula $f_{A}(x)=\langle x, A\rangle$ where $\langle-,-\rangle$ denotes the Killing form. For the proof of the following theorem see [4], [12], and $[3]$.

Theorem 1. $f_{A}: G \cdot x_{0} \rightarrow \mathbb{R}$ is a Morse-Bott function for any $A \in \mathfrak{g}$. For a generic $A$, i.e. when $A$ lies in an orbit of maximal dimension, $f_{A}$ is a Morse function.

The following claim can be established by elementary means (see for instance [18] or [5]).

\section{Claim 2.}

1. For all $x \in G \cdot x_{0}$, the tangent space to the orbit at $x$ is $T_{x}\left(G \cdot x_{0}\right)=[\mathfrak{g}, x]$.

2. For all $x \in G \cdot x_{0}$, the normal space to the orbit at $x$ is $N_{x}\left(G \cdot x_{0}\right)=\{W \in$ $\mathfrak{g} \mid[W, x]=0\}$.

3. For any $x \in G \cdot x_{0}$ and for all $X \in T_{x}\left(G \cdot x_{0}\right)$ the differential of $f_{A}$ is given by $D_{X} f_{A}=\langle X, A\rangle$.

4. $x_{c} \in G \cdot x_{0}$ is a critical point of $f_{A}$ if and only if $\left[A, x_{c}\right]=0$.

If $G$ is a matrix group, e.g. $U_{n}, O_{n}$, or $S p_{n}$, then the above Morse-Bott functions are compatible with an increase in dimension, e.g. $U_{n} \hookrightarrow U_{n+1}$, and so they can be used to define Morse-Bott functions on various orbits which arise as direct limits of matrix groups. Since this paper is mainly concerned with complex Grassmann manifolds, we will now restrict to the case where $G$ is a unitary group. For any $n, k \in \mathbb{N}$ we choose

$$
x_{0}=i\left(\begin{array}{cc}
1_{n \times n} & 0 \\
0 & 0_{k \times k}
\end{array}\right) \in u(n+k)
$$

and we see that $G_{n, n+k}(\mathbb{C})$, the complex Grassmann manifold consisting of $n$-planes in $\mathbb{C}^{n+k}$, is diffeomorphic to the orbit $U_{n+k} \cdot x_{0} \subseteq u(n+k)$ since the action of $U_{n+k}$ on both spaces is transitive with stabilizer $U_{n} \times U_{k}$, i.e. both spaces are diffeomorphic to $U_{n+k} / U_{n} \times U_{k}$. From now on we will identify $G_{n, n+k}(\mathbb{C})$ with $U_{n+k} \cdot x_{0} \subseteq u(n+k)$ by choosing the unique equivariant diffeomorphism which sends $x_{0}$ to $\mathbb{C}^{n} \subseteq \mathbb{C}^{n+k}$. This amounts to choosing a specific embedding of $G_{n, n+k}(\mathbb{C})$ into the $(n+k)^{2}$ dimensional Euclidean space $u(n+k)$ where the inner product is the Killing form.

Theorem 3. Let $p \in G_{n, n+k}(\mathbb{C})$ be any plane. For any matrix $A \in u(n+k)$ the gradient flow line of the Morse-Bott function $f_{A}$ through $p$, with respect to the pullback of the Killing form, is $\gamma_{p}(t)=L_{t}(p)$, where $L_{t}: \mathbb{C}^{n+k} \rightarrow \mathbb{C}^{n+k}$ is the linear map determined by the matrix $\exp (-i t A) \in G L_{n+k}(\mathbb{C})$ using column orientation for $\mathbb{C}^{n+k}$.

Note that if we use row orientation for $\mathbb{C}^{n+k}$, then the gradient flow is given by the linear map determined by the transpose of $\exp (-i t A)$.

We will use the above theorem to prove that $f_{A}$ satisfies the Morse-Bott-Smale transversality condition. This transversality condition can be stated as follows (see for instance [2]).

Definition 4. A function satisfies the Morse-Bott-Smale transversality condition if and only if for any two critical submanifolds $M$ and $N$ the stable manifold of $N$ 
intersects the unstable manifold of $m$ transversely for all $m \in M$, i.e. $W^{u}(m) \pitchfork$ $W^{s}(N)$ for all $m \in M$.

Theorem 5. For any $A \in u(n+k)$ the function $f_{A}: G_{n, n+k}(\mathbb{C}) \rightarrow \mathbb{R}$ satisfies the Morse-Bott-Smale transversality condition with respect to the pullback of the Killing form.

For the proof of Theorem 3 we will need the following elementary facts.

\section{Claim 6.}

1. For all $A \in u(n+k)$ and for all $x \in U_{n+k} \cdot x_{0}$ the projection of $A$ onto $T_{x}\left(U_{n+k} \cdot x_{0}\right)$ is $-[[A, x], x]$.

2. The gradient vector field of $f_{A}: U_{n+k} \cdot x_{0} \rightarrow \mathbb{R}$ with respect to the Killing form is $\left(\nabla f_{A}\right)_{x}=-[[A, x], x]$.

Proof. Let $A \in u(n+k)$ and $x \in U_{n+k} \cdot x_{0}$. The projection of $A$ onto $T_{x}\left(U_{n+k} \cdot x_{0}\right)$ is the unique vector $X \in T_{x}\left(U_{n+k} \cdot x_{0}\right)$ such that $A-X \in N_{x}\left(U_{n+k} \cdot x_{0}\right)$. That is, $[A-X, x]=0$ (see Claim 2) or equivalently, $[A, x]=[X, x]$. So the first part of the claim is simply the statement that $[A, x]=-[[[A, x], x], x]$; i.e. $[-, x]$ is an almost complex structure on $U_{n+k} \cdot x_{0}$ (see for instance [1] p. 57).

To prove the second part of the claim recall that $\left(\nabla f_{A}\right)_{x}$ is the unique element of $T_{x}\left(U_{n+k} \cdot x_{0}\right)$ which satisfies $\left\langle\left(\nabla f_{A}\right)_{x}, X\right\rangle=D_{X} f_{A}$ for all $X \in T_{x}\left(U_{n+k} \cdot x_{0}\right)$. So we have by Claim $2\left\langle\left(\nabla f_{A}\right)_{x}, X\right\rangle=\langle A, X\rangle$ for all $X \in T_{x}\left(U_{n+k} \cdot x_{0}\right)$. That is, $\left(\nabla f_{A}\right)_{x}$ is the projection of $A$ onto the tangent space $T_{x}\left(U_{n+k} \cdot x_{0}\right)$.

Lemma 7. Let $\eta: G \times M \rightarrow M$ be a smooth action of a Lie group $G$ on a smooth manifold $M$. Let $m \in M$ and let $\eta_{m}: G \rightarrow M$ be the obvious map induced from $\eta$. Then $T_{e}\left(G_{m}\right) \subseteq \operatorname{ker} d \eta_{m}(e)$, where $e \in G$ is the identity and $G_{m}$ is the stabilizer of m. Moreover, $T_{e}\left(G_{g \cdot m}\right)=\operatorname{Ad}_{g}\left(T_{e}\left(G_{m}\right)\right)$ for all $g \in G$.

Proof. Let $\gamma(t)$ be a path in $G_{m}$ with $\gamma(0)=e$. Then $\eta_{m}(\gamma(t))$ is constant and so $\left.\frac{d}{d t}\right|_{t=0} \eta_{m}(\gamma(t))=d \eta_{m}(e)\left(\gamma^{\prime}(0)\right)=0$. This shows that $T_{e}\left(G_{m}\right) \subseteq \operatorname{ker} d \eta_{m}(e)$. To prove the second statement one need merely recall that $G_{g \cdot m}=g G_{m} g^{-1}$ and then consider paths of the form $g \sigma(t) g^{-1}$, where $\sigma(t) \in G_{m}$ and $\sigma(0)=e$.

Proof of Theorem 3. Let $\mathbb{C}^{n} \subseteq \mathbb{C}^{n+k}$ be the vector subspace spanned by the first $n$ standard basis vectors of $\mathbb{C}^{n+k}$. Pick any unitary matrix $U \in U_{n+k}$ such that $U\left(\mathbb{C}^{n}\right)=p$ and let $x=U x_{0} U^{-1} \in u(n+k)$. The path $\sigma_{x}(t)=\exp (-t[A, x]) \cdot x$ $\in u(n+k)$ satisfies $\sigma_{x}^{\prime}(0)=-[[A, x], x]$, the gradient vector of $f_{A}$ at $x$ (see p. 115 of [18]). Notice that under the diffeomorphism $U_{n+k} \cdot x_{0} \approx G_{n, n+k}(\mathbb{C})$ this path goes to the path $\tilde{\sigma}_{x}(t)=\exp (-t[A, x])(p)$. Because of this and the fact that $\gamma_{p}\left(t+t_{0}\right)=\gamma_{\tilde{p}}(t)$ for some $\tilde{p} \in G_{n, n+k}(\mathbb{C})$, it suffices to show that $\gamma_{p}^{\prime}(0)=\tilde{\sigma}_{x}^{\prime}(0)$.

Let $\eta: G L_{n+k}(\mathbb{C}) \times G_{n, n+k}(\mathbb{C}) \rightarrow G_{n, n+k}(\mathbb{C})$ be the smooth action of $G L_{n+k}(\mathbb{C})$ on $G_{n, n+k}(\mathbb{C})$ given by treating an element of $G L_{n+k}(\mathbb{C})$ as a linear map and evaluating it on a plane, and let $\eta_{p}: G L_{n+k}(\mathbb{C}) \rightarrow G_{n, n+k}(\mathbb{C})$ be induced from $\eta$. In this notation $\gamma_{p}(t)=\eta_{p}(\exp (-i t A))$ and $\tilde{\sigma}_{x}(t)=\eta_{p}(\exp (-t[A, x]))$. By the chain rule we have $\gamma_{p}^{\prime}(0)=d \eta_{p}\left(I_{n+k}\right)(-i A)$ and $\tilde{\sigma}_{x}^{\prime}(0)=d \eta_{p}\left(I_{n+k}\right)(-[A, x])$, where $I_{n+k}$ is the identity matrix of size $(n+k) \times(n+k)$. Thus we will be done once we show that $d \eta_{p}\left(I_{n+k}\right)(-i A+[A, x])=0$.

To show that $-i A+[A, x] \in \operatorname{ker} d \eta_{p}\left(I_{n+k}\right)$ we apply the previous lemma and show that

$$
-i A+[A, x] \in T_{I_{n+k}}\left(G L_{n+k}(\mathbb{C})_{p}\right)=U\left(T_{I_{n+k}}\left(G L_{n+k}(\mathbb{C})_{\mathbb{C}^{n}}\right)\right) U^{-1} .
$$


If we use column orientation for $\mathbb{C}^{n+k}$, then the stabilizer of $\mathbb{C}^{n} \in G_{n, n+k}(\mathbb{C})$ consists of those elements of $G L_{n+k}(\mathbb{C})$ whose lower left $k \times n$ block is zero, and since $G L_{n+k}(\mathbb{C})$ is an open subset of $\mathbb{C}^{(n+k)^{2}}$ the tangent space at $I_{n+k}$ of the stabilizer of $\mathbb{C}^{n}$ consists of those matrices in the tangent space whose lower left $k \times n$ block is zero. Therefore all we need to do is to write $-i A+[A, x]$ as such a matrix conjugated with $U$ :

$$
\begin{aligned}
-i A+[A, x] & =-i A+\left[A, U x_{0} U^{-1}\right] \\
& =-i A+U\left[U^{-1} A U, x_{0}\right] U^{-1} \\
& =U\left(-i U^{-1} A U+\left[U^{-1} A U, x_{0}\right]\right) U^{-1}
\end{aligned}
$$

Letting $Y=U^{-1} A U$, one easily sees that $-i Y+\left[Y, x_{0}\right]$ does have its lower left $k \times n$ block identically zero.

Proof of Theorem 5. Let $A \in u(n+k)$. Since the Killing form is invariant under the adjoint action and every skew-Hermitian matrix can be conjugated by a unitary matrix to a diagonal matrix, we may assume that $A$ is diagonal. Moreover, by reordering the basis elements if necessary we may assume that the eigenvalues of $A$ (divided by $i$ ) are increasing. The matrix $\exp (-i t A)$ is then a diagonal matrix whose diagonal entries are all of the form $e^{a_{j} t}$, where $a_{j} \in \mathbb{R}$ and $a_{j} \leq a_{j+1}$ for all $1 \leq$ $j \leq n+k$. Let $V_{n, n+k}(\mathbb{C})$ be the complex Stiefel manifold. Then $\pi: V_{n, n+k}(\mathbb{C}) \rightarrow$ $G_{n, n+k}(\mathbb{C})$ is a smooth fiber bundle. If we think of an element of $V_{n, n+k}(\mathbb{C})$ as an $(n+k) \times n$ matrix of complex numbers, then $\exp (-i t A)$ acts on $V_{n, n+k}(\mathbb{C})$ by multiplication on the left. This gives a flow on $V_{n, n+k}(\mathbb{C})$, which commutes with the projection $\pi$ since $G L_{n}(\mathbb{C})$ acts on $V_{n, n+k}(\mathbb{C})$ by multiplication on the right (using column orientation for $\left.\mathbb{C}^{n+k}\right)$ to give the quotient space $V_{n, n+k}(\mathbb{C}) / G L_{n}(\mathbb{C}) \approx$ $G_{n, n+k}(\mathbb{C})$.

Let $M$ and $N$ be critical submanifolds of $G_{n, n+k}(\mathbb{C})$ such that there exists a flow from $M$ to $N$. Let $m \in M$ and choose $n \in N$ such that there is a flow from $m$ to $n$. Let $\tilde{m}, \tilde{n} \in V_{n, n+k}$ be the matrices in reduced column echelon form such that $\pi(\tilde{m})=m, \pi(\tilde{n})=n$ and the first non-zero entry in each column of $m$ and $n$ is 1 . Since $\exp (-i t A)$ is diagonal with its eigenvalues increasing, it is easy to see that $\pi^{-1}\left(W^{u}(m)\right)$ contains all matrices in $V_{n, n+k}(\mathbb{C})$ whose topmost non-zero entries in column $j$ agree with all the non-zero entries in column $j$ of $\tilde{m}$ for $j=1, \ldots, n$. Similarly, $\pi^{-1}\left(W^{s}(n)\right)$ contains all matrices in $V_{n, n+k}(\mathbb{C})$ whose bottommost non-zero entries in column $j$ agree with all the non-zero entries in column $j$ of $\tilde{n}$ for $j=1, \ldots, n$. Hence, $\pi^{-1}\left(W^{u}(m)\right) \pitchfork \pi^{-1}\left(W^{s}(n)\right)$, and since $\pi: V_{n, n+k}(\mathbb{C}) \rightarrow G_{n, n+k}(\mathbb{C})$ is locally trivial we have $W^{u}(m) \pitchfork W^{s}(n)$.

\section{The Floer cohomology of $f_{A}: G_{n}\left(\mathbb{C}\left[z, z^{-1}\right]\right) \rightarrow \mathbb{R}$}

Consider $\mathbb{C}\left[z, z^{-1}\right]$, the ring of Laurent polynomials. As a vector space $\mathbb{C}\left[z, z^{-1}\right]$ is isomorphic to $\mathbb{C}^{\infty}$ and so the Grassmann manifold $G_{n}\left(\mathbb{C}\left[z, z^{-1}\right]\right)$, which consists of all $n$-dimensional planes in $\mathbb{C}\left[z, z^{-1}\right]$, is homeomorphic to $G_{n}\left(\mathbb{C}^{\infty}\right)$. In order to put a smooth structure on $G_{n}\left(\mathbb{C}\left[z, z^{-1}\right]\right)$ we write it as a direct limit as follows.

Let $\Lambda$ denote the set of all finite subsets of $\mathbb{Z}$. That is, $\Lambda=\{\lambda \in \mathcal{P}(\mathbb{Z})|| \lambda \mid<$ $\infty\}$. $\Lambda$ is a directed set whose partial ordering is given by inclusion $\subseteq$. For any $\alpha \in \Lambda$ let $\mathbb{C}\left[z, z^{-1}\right]^{\alpha} \subseteq \mathbb{C}\left[z, z^{-1}\right]$ be the vector space spanned by $\left\{z^{j}\right\}_{j \in \alpha}$. Let $\alpha, \beta \in \Lambda$ with $\alpha \subseteq \beta$. Then $\mathbb{C}\left[z, z^{-1}\right]^{\alpha} \subseteq \mathbb{C}\left[z, z^{-1}\right]^{\beta}$ induces an inclusion $j_{\alpha}^{\beta}$ : 
$G_{n}\left(\mathbb{C}\left[z, z^{-1}\right]^{\alpha}\right) \hookrightarrow G_{n}\left(\mathbb{C}\left[z, z^{-1}\right]^{\beta}\right)$ which sends an $n$-plane in $\mathbb{C}\left[z, z^{-1}\right]^{\alpha}$ to itself viewed as a plane in $\mathbb{C}\left[z, z^{-1}\right]^{\beta}$. Thus $\left\{G_{n}\left(\mathbb{C}\left[z, z^{-1}\right]^{\alpha}\right), j_{\alpha}^{\beta}\right\}_{\alpha, \beta \in \Lambda}$ is a direct system of smooth manifolds whose direct limit is $G_{n}\left(\mathbb{C}\left[z, z^{-1}\right]\right)$. This describes the smooth structure on $G_{n}\left(\mathbb{C}\left[z, z^{-1}\right]\right)$. Note that $G_{n}\left(\mathbb{C}\left[z, z^{-1}\right]\right)$ is a smooth manifold modeled on the space $\bigoplus_{j=-\infty}^{\infty} \mathbb{C}$. In particular, $G_{n}\left(\mathbb{C}\left[z, z^{-1}\right]\right)$ is not a Banach manifold.

We can also use the indexing set $\Lambda$ to define direct systems of unitary groups and their Lie algebras. Fix any $\lambda \in \Lambda$ and let $U(\lambda)$ denote the unitary group of $\mathbb{C}\left[z, z^{-1}\right]^{\lambda}$. Notice that the indexing set for the matrix entries is $\lambda$. This gives for all $\alpha, \beta \in \Lambda$ with $\alpha \leq \beta$ an inclusion $U(\alpha) \subseteq U(\beta)$ given by $\left(a_{r s}\right) \mapsto\left(b_{r s}\right)$, where

$$
b_{r s}= \begin{cases}a_{r s} & \text { if } r, s \in \alpha, \\ 0 & \text { if } r \text { or } s \notin \alpha \text { and } r \neq s \\ 1 & \text { if } r \text { or } s \notin \alpha \text { and } r=s .\end{cases}
$$

In terms of maps this inclusion sends a unitary map $U_{\alpha} \in U(\alpha)$ to the unitary map $U_{\beta} \in U(\beta)$ which acts on $\mathbb{C}\left[z, z^{-1}\right]^{\alpha} \subseteq \mathbb{C}\left[z, z^{-1}\right]^{\beta}$ the same way $U_{\alpha}$ acts on $\mathbb{C}\left[z, z^{-1}\right]^{\alpha}$ and which fixes $\mathbb{C}\left[z, z^{-1}\right]^{\beta} \backslash \mathbb{C}\left[z, z^{-1}\right]^{\alpha}$. Similarly for any $\lambda \in \Lambda$ we define the Lie algebra of skew-Hermitian matrices $u(\lambda)$ to be the associated Lie algebra where the matrix entries are indexed by $\lambda$.

If for every $\lambda \in \Lambda$ we choose a skew-Hermitian matrix $A_{\lambda} \in u(\lambda)$ such that $j_{\alpha}^{\beta}\left(A_{\alpha}\right)=A_{\beta}$ for every $\alpha, \beta \in \Lambda$, then we can define functions $f_{A_{\lambda}}(x)=\left\langle x, A_{\lambda}\right\rangle$ such that $f_{A_{\beta}} \circ j_{\alpha}^{\beta}=f_{A_{\alpha}}$. The functions $f_{A_{\lambda}}$ are all smooth and hence in the limit define a smooth function $f_{A}: G_{n}\left(\mathbb{C}\left[z, z^{-1}\right]\right) \rightarrow \mathbb{R}$. Since the Killing form is compatible with the inclusions $j_{\alpha}^{\beta}$ the gradient flow lines of $f_{A_{\alpha}}$ get mapped to gradient flow lines of $f_{A_{\beta}}$ under $j_{\alpha}^{\beta}$, and so it makes sense to talk about the gradient flow lines of $f_{A}$.

We now choose a set of generic skew-Hermitian matrices $A_{\lambda} \in u(\lambda)$ and compute the Floer cohomology of the function $f_{A}: G_{n}\left(\mathbb{C}\left[z, z^{-1}\right]\right) \rightarrow \mathbb{R}$. A matrix $A_{\lambda} \in u(\lambda)$ is generic if and only if it has distinct eigenvalues. By Theorem $1, f_{A_{\lambda}}$ will have discrete critical points in this case. For the sake of exposition we will from now on limit ourselves to indexing sets $\lambda \in \Lambda$ of the form

$$
\lambda=\left\{m_{1}, m_{1}+1, m_{1}+2, \ldots, m_{2}-1, m_{2}\right\}
$$

for some $m_{1}, m_{2} \in \mathbb{Z}$ with $m_{1}<m_{2}$. For every such $\lambda \in \Lambda$ we let $A_{\lambda} \in u(\lambda)$ be the skew-Hermitian matrix whose $m$ th diagonal entry is $-i m$ for every $m \in \lambda$ and all its other entries are zero. We define $f_{A}=\lim f_{A_{\lambda}}$. Part 4 of Claim 2 implies that the critical points of $f_{A_{\lambda}}$ are the diagonal matrices in the orbit. Since conjugation by a unitary matrix does not change the eigenvalues, the critical points of $f_{A_{\lambda}}$ all have exactly $n$ square roots of minus one along the diagonal and all other entries zero. We denote these critical points by $x_{\sigma}$, where $\sigma=\left(r_{1}, \ldots, r_{n}\right)$ is an $n$-tuple of integers with $r_{j} \in \lambda$ for all $j=1, \ldots, n$ and $r_{1}<r_{2}<\cdots<r_{n} . x_{\sigma}$ is the matrix with $i$ in its $\left(r_{j}, r_{j}\right)$ entry for all $j=1, \ldots, n$ and 0 in all its other entries. (Recall that we are indexing the entries of the matrices in $u(\lambda)$ by $\lambda$.) The corresponding point of $G_{n}\left(\mathbb{C}\left[z, z^{-1}\right]\right)$ will be denoted by $p_{\sigma}$.

We now describe the unstable manifolds $W^{u}\left(p_{\sigma}\right)$ of $f_{A_{\lambda}}$. Given any Schubert symbol $\sigma=\left(r_{1}, \ldots, r_{n}\right)$, we recall (see [15], pp. 74-75) that the associated Schubert cell $e(\sigma) \subseteq G_{n}\left(\mathbb{C}\left[z, z^{-1}\right]^{\lambda}\right)$ is defined to be the set of all planes $p \in G_{n}\left(\mathbb{C}\left[z, z^{-1}\right]^{\lambda}\right)$ such that

$$
\operatorname{dim}\left(p \cap \mathbb{C}\left[z, z^{-1}\right]^{\left\{r_{1}, r_{1}+1, \ldots, r_{j}\right\}}\right)=j
$$


and

$$
\operatorname{dim}\left(p \cap \mathbb{C}\left[z, z^{-1}\right]^{\left\{r_{1}, r_{1}+1, \ldots, r_{j}-1\right\}}\right)=j-1
$$

for all $j=1, \ldots, n$. The following theorem relates the unstable manifolds of $f_{A_{\lambda}}$ to the Schubert cells of $G_{n}\left(\mathbb{C}\left[z, z^{-1}\right]^{\lambda}\right)$.

Theorem 8. For any Schubert symbol $\sigma=\left(r_{1}, \ldots, r_{n}\right)$,

$$
W^{u}\left(p_{\sigma}\right)=e(\sigma)
$$

Proof. Let $\sigma=\left(r_{1}, \ldots, r_{n}\right)$ be any Schubert symbol and let $p \in e(\sigma)$. We must show that for any open neighborhood $U \in G_{n}\left(\mathbb{C}\left[z, z^{-1}\right]^{\lambda}\right)$ containing $p_{\sigma}$, there exists $T<0$ such that for all $t<T$ we have $\gamma_{p}(t)=\exp (-i t A)(p) \in U$. Since $p \in e(\sigma)$ we can pick a basis $v_{1}, \ldots, v_{n}$ of $p$ such that for $j=1, \ldots, n$ the vector $v_{j}$ has 1 in the $r_{j}$ th entry and 0 in all entries below $r_{j}$. The vectors

$$
e^{r_{1} t} \exp (-i t A)\left(v_{1}\right), \ldots, e^{r_{n} t} \exp (-i t A)\left(v_{n}\right)
$$

span the plane $\exp (-i t A)(p)$. Note that for $j=1, \ldots, n$ the entries of the vector $e^{r_{j} t} \exp (-i t A)\left(v_{j}\right)$ are as follows:

$$
m \text { th entry of } e^{r_{j} t} \exp (-i t A)\left(v_{j}\right)= \begin{cases}z e^{\left(r_{j}-m\right) t} \text { some } z \in \mathbb{C} & \text { if } m<r_{j}, \\ 1 & \text { if } m=r_{j}, \\ 0 & \text { if } m>r_{j}\end{cases}
$$

If we consider $\tilde{\gamma}_{p}(t)=\left(e^{r_{1} t} \exp (-i t A)\left(v_{1}\right), \ldots, e^{r_{n} t} \exp (-i t A)\left(v_{n}\right)\right)$ as a path in $V_{n}\left(\mathbb{C}\left[z, z^{-1}\right]^{\lambda}\right)$, then we have $\pi\left(\tilde{\gamma}_{p}(t)\right)=\gamma_{p}(t)$ for all $t \in \mathbb{R}$. Since $\left(e_{r_{1}}, \ldots, e_{r_{n}}\right) \in$ $\pi^{-1}(U)$ where $\pi^{-1}(U)$ is open in $V_{n}\left(\mathbb{C}\left[z, z^{-1}\right]^{\lambda}\right)$ and $\lim _{t \rightarrow-\infty} \tilde{\gamma}_{p}(t)=\left(e_{r_{1}}, \ldots, e_{r_{n}}\right)$, we can choose a $T<0$ such that for all $t<T$ we have $\tilde{\gamma}_{p}(t) \in \pi^{-1}(U)$. But this implies that $\gamma_{p}(t) \in U$ for all $t<T$.

We have shown that $e(\sigma) \subseteq W^{u}\left(p_{\sigma}\right)$. Now let $p \in W^{u}\left(p_{\sigma}\right)$. Since the Schubert cells partition $G_{n}\left(\mathbb{C}\left[z, z^{-1}\right]^{\lambda}\right)$, there exists a unique a Schubert symbol $\tilde{\sigma}$ such that $p \in e(\tilde{\sigma}) \subseteq W^{u}\left(p_{\tilde{\sigma}}\right)$. But then $p \in W^{u}\left(p_{\tilde{\sigma}}\right)$. Since the unstable manifolds are disjoint, this says that $\tilde{\sigma}=\sigma$, and so $p \in e(\sigma)$.

Recalling that for any critical point $p_{\sigma}$ the unstable manifold theorem says that $W^{u}\left(p_{\sigma}\right)$ is an embedded disk whose dimension is equal to the index of $p_{\sigma}$, we have the following.

Corollary 9. The index of $p_{\sigma}$ in $G_{n}\left(\mathbb{C}\left[z, z^{-1}\right]^{\lambda}\right)$ can be computed by adding up the number of rows of $x_{\sigma} \in u(\sigma)$ above each $i$ which consist entirely of zeros and then multiplying by 2.

The preceding corollary shows that the index of a critical point is not preserved under the inclusions $j_{\alpha}^{\beta}: G_{n}\left(\mathbb{C}\left[z, z^{-1}\right]^{\alpha}\right) \rightarrow G_{n}\left(\mathbb{C}\left[z, z^{-1}\right]^{\beta}\right)$. However, the relative index of critical points is preserved under the inclusion $j_{\alpha}^{\beta}: G_{n}\left(\mathbb{C}\left[z, z^{-1}\right]^{\alpha}\right) \rightarrow$ $G_{n}\left(\mathbb{C}\left[z, z^{-1}\right]^{\beta}\right)$, where $\alpha$ is in the cofinal system consisting of those sets which include all integers between the smallest and the largest in the set. That is, if $p_{\sigma}$ and $p_{\tilde{\sigma}}$ are any two critical points in $G_{n}\left(\mathbb{C}\left[z, z^{-1}\right]^{\alpha}\right)$ where $\alpha=\left\{m_{1}, m_{1}+1, \ldots, m_{2}\right\}$ for some $m_{1}, m_{2} \in \mathbb{Z}$ with $m_{1}<m_{2}$, then for all $\beta \in \Lambda$

$$
\operatorname{index}\left(p_{\sigma}\right)-\operatorname{index}\left(p_{\tilde{\sigma}}\right)=\operatorname{index}\left(j_{\alpha}^{\beta}\left(p_{\sigma}\right)\right)-\operatorname{index}\left(j_{\alpha}^{\beta}\left(p_{\tilde{\sigma}}\right)\right) .
$$

This allows us to define a relative index between critical points of $f_{A}: G_{n}\left(\mathbb{C}\left[z, z^{-1}\right]\right)$ $\rightarrow \mathbb{R}$ analogous to Floer's relative index for critical points of the action functional (see for instance [11], p. 584). 
Definition 10. Let $p_{\sigma}, p_{\tilde{\sigma}} \in G_{n}\left(\mathbb{C}\left[z, z^{-1}\right]\right)$ be critical points of the function $f_{A}$ : $G_{n}\left(\mathbb{C}\left[z, z^{-1}\right]\right) \rightarrow \mathbb{R}$. Let $p_{\sigma^{\prime}}$ be any critical point of $f_{A}$ such that $p_{\sigma^{\prime}} \leq p_{\sigma}$ and $p_{\sigma^{\prime}} \leq p_{\tilde{\sigma}}$. The relative index of $p_{\sigma}$ and $p_{\tilde{\sigma}}$ is defined to be

$$
\operatorname{ind}\left(p_{\sigma}, p_{\tilde{\sigma}}\right)=\operatorname{dim} W\left(p_{\sigma}, p_{\sigma^{\prime}}\right)-\operatorname{dim} W\left(p_{\tilde{\sigma}}, p_{\sigma^{\prime}}\right),
$$

where $W(a, b)=W^{u}(a) \cap W^{s}(b)$ for any two critical points a and $b$.

We define the Floer chain complex $\mathcal{C}_{*}$ as follows. Fix any critical point $p_{0}$ and for each $j \in \mathbb{Z}$ let $C_{j}$ be the free abelian group on the critical points $p_{\sigma}$ such that $\operatorname{ind}\left(p_{\sigma}, p_{0}\right)=j$. The Floer chain complex is then defined to be:

$$
\cdots \rightarrow C_{2} \rightarrow C_{1} \rightarrow C_{0} \rightarrow C_{-1} \rightarrow C_{-2} \rightarrow \cdots,
$$

where the boundary homomorphisms are given on the generators by counting (with sign) the number of gradient flow lines between two critical points of relative index 1. In this case all the boundary homomorphisms are zero, since $C_{j}=0$ for $j$ odd. Note that the Floer chain complex is independent of the choice of base point up to degree shift. The homology of this complex is $\bigoplus_{j=1}^{\infty} \mathbb{Z}$ in all even dimensions (positive and negative) and 0 in all odd dimensions. For the sake of exposition we will now fix $p_{0}=p_{(0,1, \ldots, n-1)}$.

We describe the module structure of $H F^{*}\left(G_{n}\left(\mathbb{C}\left[z, z^{-1}\right]\right)\right)$ over $H^{*}\left(G_{n}\left(\mathbb{C}^{\infty}\right)\right)$. The filtration

$$
\mathbb{C}\left[z, z^{-1}\right]^{\{0,1, \ldots\}} \subseteq \mathbb{C}\left[z, z^{-1}\right]^{\{-1,0, \ldots\}} \subseteq \mathbb{C}\left[z, z^{-1}\right]^{\{-2,-1, \ldots\}} \ldots \subseteq \mathbb{C}\left[z, z^{-1}\right]
$$

induces a filtration of the Floer chain complex $\mathcal{C}_{*}$ as follows. For each $N \in \mathbb{Z}_{+}$we have

$$
G_{n}\left(\mathbb{C}\left[z, z^{-1}\right]^{\{-N,-N+1, \ldots\}}\right) \subseteq G_{n}\left(\mathbb{C}\left[z, z^{-1}\right]\right),
$$

and we let $\left.\mathcal{C}_{*}\right|_{-N} \subseteq \mathcal{C}_{*}$ be the chain complex generated by the critical points which lie in $G_{n}\left(\mathbb{C}\left[z, z^{-1}\right]^{\{-N,-N+1, \ldots\}}\right)$. We define the dual of this filtered complex to be $\left.\mathcal{C}^{*}\right|_{-N}=\operatorname{Hom}\left(\left.\mathcal{C}_{*}\right|_{-N}, \mathbb{Z}\right)$. Note that $\left.\mathcal{C}^{*}\right|_{-N}$ is the dual of the Morse complex for $f_{A}$ restricted to $G_{n}\left(\mathbb{C}\left[z, z^{-1}\right]^{\{-N,-N+1, \ldots\}}\right)$ but with the groups shifted down $2 N n$ dimensions. In particular, $H^{*}\left(\left.\mathcal{C}^{*}\right|_{-N}\right)$ is $\mathbb{Z}$ in dimension $-2 N n$, and all the groups below dimension $-2 N n$ are zero.

Definition 11. The Floer cohomology of $f_{A}: G_{n}\left(\mathbb{C}\left[z, z^{-1}\right]\right) \rightarrow \mathbb{R}$ as a module over $H^{*}\left(G_{n}\left(\mathbb{C}^{\infty}\right)\right)$ is defined to be the following direct limit:

$$
H F^{*}\left(G_{n}\left(\mathbb{C}\left[z, z^{-1}\right]\right)\right)=\lim _{\vec{N}} H^{*}\left(\left.\mathcal{C}^{*}\right|_{-N}\right),
$$

where the maps in the direct system are given by identifying each term in the limit with the dual of the Morse complex of $f_{A}$ restricted to $G_{n}\left(\mathbb{C}\left[z, z^{-1}\right]^{\{-N,-N+1, \ldots\}}\right)$.

In order to make the isomorphisms in the preceding definition explicit we define a degree $2 n$ chain homomorphism $S_{1}: \mathcal{C}_{*} \rightarrow \mathcal{C}_{*+2 n}$ by $S_{1}\left(p_{\left(r_{1}, \ldots, r_{n}\right)}\right)=p_{\left(r_{1}+1, \ldots, r_{n}+1\right)}$. $S_{1}$ sends a critical point of relative index $j$ to a critical point of relative index $j+2 n$, and for all $j \in \mathbb{Z}$ it induces an isomorphism

$$
H_{j}\left(\left.\mathcal{C}_{*}\right|_{-1}\right) \stackrel{S_{1}}{\rightarrow} H_{j+2 n}\left(\left.\mathcal{C}_{*}\right|_{0}\right) \approx H_{j+2 n}\left(G_{n}\left(\mathbb{C}\left[z, z^{-1}\right]^{\{-1,0, \ldots\}}\right)\right) .
$$

For all $j \in \mathbb{Z}$ we define $p_{\left(r_{1}, \ldots, r_{n}\right)}^{*} \in \operatorname{Hom}\left(\mathcal{C}_{j}, \mathbb{Z}\right)$ to be the homomorphism which takes the value 1 on $p_{\left(r_{1}, \ldots, r_{n}\right)} \in \mathcal{C}_{j}$ and 0 on all other critical points. $\left.\mathcal{C}^{j}\right|_{-N}$ is the free abelian group on all $p_{\left(r_{1}, \ldots, r_{n}\right)}^{*}$ such that $p_{\left(r_{1}, \ldots, r_{n}\right)} \in G_{n}\left(\mathbb{C}\left[z, z^{-1}\right]^{\{-N,-N+1, \ldots\}}\right)$ 
and $\operatorname{ind}\left(p_{\left(r_{1}, \ldots, r_{n}\right)}, p_{0}\right)=j . S_{1}: \mathcal{C}_{*} \rightarrow \mathcal{C}_{*+2 n}$ induces a degree $2 n$ cochain homomorphism $S_{1}^{*}: \mathcal{C}^{*} \rightarrow \mathcal{C}^{*+2 n}$ defined by $S_{1}^{*}\left(p_{\left(r_{1}, \ldots, r_{n}\right)}^{*}\right)=p_{\left(r_{1}+1, \ldots, r_{n}+1\right)}^{*}$. For all $j \in \mathbb{Z}$, $S_{1}^{*}$ induces an isomorphism

$$
H^{j}\left(\left.\mathcal{C}^{*}\right|_{-1}\right) \stackrel{S_{1}^{*}}{\rightarrow} H^{j+2 n}\left(\left.\mathcal{C}^{*}\right|_{0}\right) \approx H^{j+2 n}\left(G_{n}\left(\mathbb{C}\left[z, z^{-1}\right]^{\{-1,0, \ldots\}}\right)\right) .
$$

Similarly, for all $N \in \mathbb{Z}$ we define a degree $2 N n$ chain homomorphism $S_{N}: \mathcal{C}_{*} \rightarrow$ $\mathcal{C}_{*+2 N n}$ by $S_{N}\left(p_{\left(r_{1}, \ldots, r_{n}\right)}\right)=p_{\left(r_{1}+N, \ldots, r_{n}+N\right)}$ and a degree $2 N n$ cochain homomor$\operatorname{phism} S_{N}^{*}: \mathcal{C}^{*} \rightarrow \mathcal{C}^{*+2 N n}$ by $S_{N}^{*}\left(p_{\left(r_{1}, \ldots, r_{n}\right)}^{*}\right)=p_{\left(r_{1}+N, \ldots, r_{n}+N\right)}^{*}$. For all $j \in \mathbb{Z}, S_{N}$ and $S_{N}^{*}$ induce isomorphisms

$$
\begin{array}{lll}
H_{j}\left(\left.\mathcal{C}_{*}\right|_{-N}\right) & \stackrel{S_{N}}{\rightarrow} & H_{j+2 N n}\left(\left.\mathcal{C}_{*}\right|_{0}\right) \approx H_{j+2 N n}\left(G_{n}\left(\mathbb{C}\left[z, z^{-1}\right]^{\{-N,-N+1, \ldots\}}\right)\right), \\
H^{j}\left(\left.\mathcal{C}^{*}\right|_{-N}\right) & \stackrel{S_{N}^{*}}{\longrightarrow} & H^{j+2 N n}\left(\left.\mathcal{C}^{*}\right|_{0}\right) \approx H^{j+2 N n}\left(G_{n}\left(\mathbb{C}\left[z, z^{-1}\right]^{\{-N,-N+1, \ldots\}}\right)\right) .
\end{array}
$$

Theorem 12. For all $N \in \mathbb{Z}_{+}$the following diagram commutes:

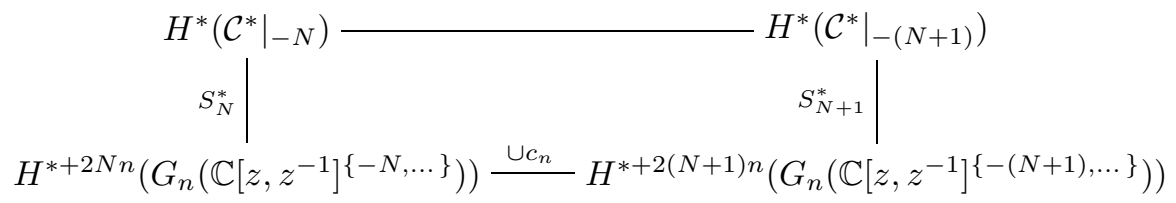

where $c_{n}$ is the Euler class of $\gamma_{n}$, the tautological n-plane bundle over $G_{n}\left(\mathbb{C}^{\infty}\right)$.

Corollary 13. The Floer cohomology of $f_{A}: G_{n}\left(\mathbb{C}\left[z, z^{-1}\right]\right) \rightarrow \mathbb{R}$ as a module over $H^{*}\left(G_{n}\left(\mathbb{C}^{\infty}\right)\right)$ is

$$
H F^{*}\left(G_{n}\left(\mathbb{C}\left[z, z^{-1}\right]\right)\right)=\lim _{\vec{N}} H^{*}\left(\left.\mathcal{C}^{*}\right|_{-N}\right) \approx H^{*}\left(G_{n}\left(\mathbb{C}^{\infty}\right)\right)\left[c_{n}^{-1}\right],
$$

where $c_{n}$ denotes the Euler class of $\gamma_{n}$, the tautological $n$-plane bundle over $G_{n}\left(\mathbb{C}^{\infty}\right)$.

Proof of Theorem 12. Recall from Theorem 8 that for all Schubert symbols $\sigma$ we have $W^{u}\left(p_{\sigma}\right)=e(\sigma)$. This makes the isomorphism $H^{*}\left(\left.\mathcal{C}^{*}\right|_{0}\right) \approx H^{*}\left(G_{n}\left(\mathbb{C}^{\infty}\right)\right)$ very explicit. The isomorphism identifies $\left[p_{\left(r_{1}, \ldots, r_{n}\right)}^{*} \in H^{*}\left(\left.\mathcal{C}^{*}\right|_{0}\right)\right.$ with the cohomology class in $H^{*}\left(G_{n}\left(\mathbb{C}^{\infty}\right)\right) \approx \operatorname{Hom}\left(H_{*}\left(G_{n}\left(\mathbb{C}^{\infty}\right)\right), \mathbb{Z}\right)$ which has value 1 on $\left[e\left(r_{1}, \ldots, r_{n}\right)\right] \in H_{*}\left(G_{n}\left(\mathbb{C}^{\infty}\right)\right)$ and is zero on all other homology classes. From this description we see that the map in question sends the cohomology class represented by the dual of $\left[e\left(r_{1}, \ldots, r_{n}\right)\right]$ to the cohomology class represented by the dual of $\left[e\left(r_{1}+1, \ldots, r_{n}+1\right)\right]$. Using the Schubert calculus one sees that this map is cupping with $c_{n}$ (see for instance [17], Th. 2.5, p. 229, and Th. 3.0, p. 230).

\section{The PRo-spectrum associated to $\mathcal{C}_{f_{A}}$}

Let $X$ be any compact space and let $\xi \in K(X)$. There exist a vector bundle $E \rightarrow X$ and a number $m \in \mathbb{Z}_{+}$such that $\xi=E-\varepsilon_{m}$, where $\varepsilon_{m}$ is the trivial bundle of dimension $m$. We define

$$
\mathbf{X}^{\xi}=S^{-m} \mathbf{X}^{\mathbf{E}}
$$

where $\mathbf{X}^{\mathbf{E}}$ is the suspension spectrum of the Thom space $X^{E}$. If $X$ is a CW complex of finite type, then (following [8], p. 322) for all $m \in \mathbb{Z}_{+}$we choose vector bundles $E_{m}$ over the $m$-skeleton $X^{(m)}$ such that $\left.\xi\right|_{X^{(m)}}=E_{m}-\varepsilon_{k_{m}} \in K\left(X^{(m)}\right)$ 
for some $k_{m} \in \mathbb{Z}_{+}$and such that for any $m_{1}, m_{2} \in \mathbb{Z}_{+}$there is a bundle map $E_{m_{1}} \oplus \varepsilon_{\left(k_{m_{2}}-k_{m_{1}}\right)} \rightarrow E_{m_{2}}$ covering the inclusion $X^{\left(m_{1}\right)} \rightarrow X^{\left(m_{2}\right)}$ which is an isomorphism on fibers. This gives for all $m \in \mathbb{Z}_{+}$maps

$$
S^{k_{m+1}-k_{m}}\left(X^{(m)}\right)^{E_{m}} \rightarrow\left(X^{(m+1)}\right)^{E_{m+1}},
$$

which together with the spaces $\left\{S^{m+|\xi|-\left|E_{m}\right|}\left(X^{(m)}\right)^{E_{m}}\right\}$ define the Thom spectrum $\mathbf{X}^{\xi}$. This Thom spectrum is independent of the choices made, up to homotopy.

Now consider the following situation for $X$ a compact topological space. Let $\eta, \zeta$ and $\xi$ be complex vector bundles over $X$ with $\eta \oplus \zeta=\xi$. Then the inclusion $E(\eta) \subseteq E(\xi)$ induces a map $X^{\eta} \rightarrow X^{\xi}$. Now assume $\eta, \xi \in K(X)$. Then we get a similar map as follows. Let $\eta=F-\varepsilon_{m}$ and $\xi=F \oplus \zeta-\varepsilon_{m}$. (We are still assuming that $\zeta$ is a vector bundle.) By definition we have

$$
\mathbf{X}^{\xi}=S^{-m}\left(\mathbf{X}^{\mathbf{F}+\zeta}\right) \text { and } \mathbf{X}^{\eta}=S^{-m}\left(\mathbf{X}^{\mathbf{F}}\right),
$$

and so we have an induced map $\mathbf{X}^{\eta} \rightarrow \mathbf{X}^{\xi}$. For any vector bundle $E \rightarrow X$ and for any $N \in \mathbb{Z}_{+}$we have $-(N+1) E+E=-N E$. This defines a map

$$
\mathbf{X}^{-\mathbf{N E}} \leftarrow \mathbf{X}^{-(\mathbf{N}+\mathbf{1}) \mathbf{E}} \text {. }
$$

A similar situation holds when $X$ is a CW complex of finite type. (See [8], pp. 320-323, for more details.)

The following theorem shows that the algebraic computations done in the previous section can be realized geometrically.

Theorem 14. For all $N \in \mathbb{Z}_{+}$the maps

$$
G_{n}\left(\mathbb{C}^{\infty}\right)^{-(N+1) \gamma_{n}} \rightarrow G_{n}\left(\mathbb{C}^{\infty}\right)^{-N \gamma_{n}}
$$

induced by $-(N+1) \gamma_{n}+\gamma_{n}=-N \gamma_{n}$ make the following diagram commute:

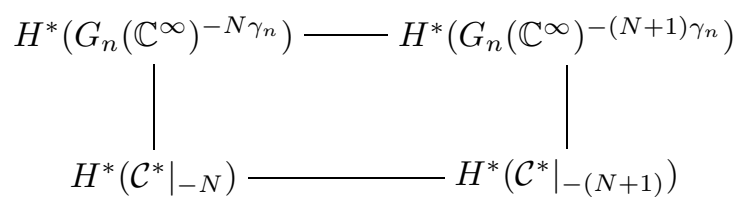

where the vertical maps are given by the Thom Isomorphism Theorem.

Proof. By Theorem 12 the following diagram commutes:

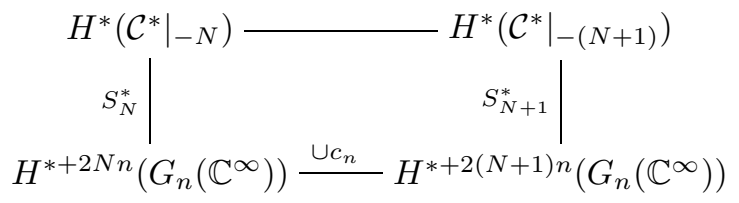

and it follows from the Thom Isomorphism Theorem that the following diagram commutes:

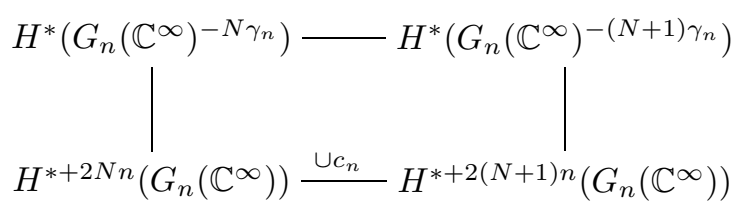


In [6] and [8] Cohen, Jones, and Segal defined the Floer homotopy type of a function such as $f_{A}: G_{n}\left(\mathbb{C}\left[z, z^{-1}\right]\right) \rightarrow \mathbb{R}$ which takes into account the above geometry. The Floer homotopy type is an inverse system of spectra constructed from the flow category of $f_{A}$ (see [7] and [13]).

Definition 15. The flow category of $f_{A}: G_{n}\left(\mathbb{C}\left[z, z^{-1}\right]\right) \rightarrow \mathbb{R}$, denoted $\mathcal{C}_{f_{A}}$, is the topological category whose objects are the critical points of $f_{A}$ topologized as a subspace of $G_{n}\left(\mathbb{C}\left[z, z^{-1}\right]\right)$ and whose morphisms are the unparameterized piecewise gradient flow lines of $f_{A}$. That is, for any two critical points a and $b, \operatorname{Mor}(a, b)$ is defined to be the space of all continuous curves $\omega:\left[f_{A}(b), f_{A}(a)\right] \rightarrow G_{n}\left(\mathbb{C}\left[z, z^{-1}\right]\right)$ satisfying

1. $\omega\left(f_{A}(b)\right)=b$,

2. $\omega\left(f_{A}(a)\right)=a$,

3. away from the critical points of $f_{A}$ the map $\omega$ is smooth and satisfies the following differential equation:

$$
\frac{d \omega}{d t}=\frac{\nabla\left(f_{A}\right)}{\left\|\nabla\left(f_{A}\right)\right\|^{2}} .
$$

$\operatorname{Mor}(a, b)$ is topologized as a subset of the space of all continuous maps from the closed interval $\left[f_{A}(b), f_{A}(a)\right]$ to $G_{n}\left(\mathbb{C}\left[z, z^{-1}\right]\right)$. This space of continuous maps is given the compact open topology.

Let $a, b \in \operatorname{Ob}\left(\mathcal{C}_{f_{A}}\right)$ with $b \leq a$, i.e. there exists a gradient flow line of $f_{A}$ beginning at $a$ and ending at $b$. We will use the notation $\left.\mathcal{C}_{f_{A}}\right|_{b} ^{a}$ to denote the full subcategory whose objects are the critical points between $a$ and $b .\left.B \mathcal{C}_{f_{A}}\right|_{b} ^{a}$ denotes the geometric realization of this restricted category. In [6] Cohen, Jones, and Segal prove the following theorem.

Theorem 16. Let $f: M \rightarrow \mathbb{R}$ be a Morse function defined on a closed Riemannian manifold $M$. Then associated to $f$ is a topological category $\mathcal{C}_{f}$ whose objects are the critical points of $f$ and whose space of morphisms between critical points a and $b$ is the space of piecewise flow lines, of the gradient flow of $f$, joining a to $b$.

(1) If $f$ is a generic Morse function (one whose gradient flow satisfies the MorseSmale transversality condition), then there is a homeomorphism

$$
B \mathcal{C}_{f} \cong M .
$$

(2) For any Morse function there is a homotopy equivalence

$$
B \mathcal{C}_{f} \simeq M .
$$

To define the Floer homotopy type of $f_{A}: G_{n}\left(\mathbb{C}\left[z, z^{-1}\right]\right) \rightarrow \mathbb{R}$ we will use the following notation. Let

$$
I=\left\{b \in \operatorname{Ob}\left(\mathcal{C}_{f_{A}}\right) \mid \overline{W\left(p_{0}, b\right)} \text { is a manifold }\right\} .
$$

For each $b \in I$ let

$$
I_{b}=\left\{a \in \operatorname{Ob}\left(\mathcal{C}_{f_{A}}\right) \mid p_{0} \leq a \text { and } \overline{W(a, b)} \text { is a manifold }\right\} .
$$

Note that for every $a \in I_{b}$ the preceding theorem says that $\left.B \mathcal{C}_{f_{A}}\right|_{b} ^{p_{0}} \cong \overline{W\left(p_{0}, b\right)}$ and $\left.B \mathcal{C}_{f_{A}}\right|_{b} ^{a} \cong \overline{W(a, b)}$, and so we have smooth structures defined on $\left.B \mathcal{C}_{f_{A}}\right|_{b} ^{p_{0}}$ and $\left.B \mathcal{C}_{f_{A}}\right|_{b} ^{a}$. For each $a \in I_{b}$ let $\nu_{a}$ be the normal bundle

$$
\left.\left.B \mathcal{C}_{f_{A}}\right|_{b} ^{p_{0}} \stackrel{\nu_{a}}{\hookrightarrow} B \mathcal{C}_{f_{A}}\right|_{b} ^{a}
$$


Note that if $a_{1}, a_{2} \in I_{b}$ with $a_{1} \leq a_{2}$, then $\nu_{a_{1}} \oplus \eta=\nu_{a_{2}}$, where $\eta$ is the normal bundle of $\left.\left.B \mathcal{C}_{f_{A}}\right|_{b} ^{a_{1}} \hookrightarrow B \mathcal{C}_{f_{A}}\right|_{b} ^{a_{2}}$ restricted to $\left.B \mathcal{C}_{f_{A}}\right|_{b} ^{p_{0}}$, and so we have a map

$$
\left(\left.B \mathcal{C}_{f_{A}}\right|_{b} ^{p_{0}}\right)^{-\nu_{a_{1}}} \leftarrow\left(\left.B \mathcal{C}_{f_{A}}\right|_{b} ^{p_{0}}\right)^{-\nu_{a_{2}}}
$$

defined. These maps define an inverse system of Thom spaces. The inverse systems $\left\{\left(\left.B \mathcal{C}_{f_{A}}\right|_{b} ^{p_{0}}\right)^{-\nu_{a}}\right\}_{a \in I_{b}}$ form a direct system as follows. For any $b_{1}, b_{2} \in I$ we say $b_{1} \lessdot b_{2}$ if and only if $\left.\left.B \mathcal{C}_{f_{A}}\right|_{b_{1}} ^{p_{0}} \subseteq B \mathcal{C}_{f_{A}}\right|_{b_{2}} ^{p_{0}}$ and $I_{b_{1}} \subseteq I_{b_{2}}$. If $b_{1} \lessdot b_{2}$ then we have an inclusion map

$$
\left\{\left(\left.B \mathcal{C}_{f_{A}}\right|_{b_{1}} ^{p_{0}}\right)^{-\nu_{a}}\right\}_{a \in I_{b_{1}}} \rightarrow\left\{\left(\left.B \mathcal{C}_{f_{A}}\right|_{b_{2}} ^{p_{0}}\right)^{-\nu_{a}}\right\}_{a \in I_{b_{2}}} .
$$

Definition 17. The Floer homotopy type of $f_{A}: G_{n}\left(\mathbb{C}\left[z, z^{-1}\right]\right) \rightarrow \mathbb{R}$ is defined to be

$$
\lim _{\overrightarrow{b \in I}}\left\{\left(\left.B \mathcal{C}_{f_{A}}\right|_{b} ^{p_{0}}\right)^{-\nu_{a}}\right\}_{a \in I_{b}}
$$

The following theorem shows how the above algebraic computation of the Floer cohomology of $f_{A}: G_{n}\left(\mathbb{C}\left[z, z^{-1}\right]\right) \rightarrow \mathbb{R}$ is reflected in its Floer homotopy type.

Theorem 18. The Floer homotopy type of $f_{A}: G_{n}\left(\mathbb{C}\left[z, z^{-1}\right]\right) \rightarrow \mathbb{R}$ has the following inverse system of spectra:

$$
G_{n}\left(\mathbb{C}^{\infty}\right) \leftarrow G_{n}\left(\mathbb{C}^{\infty}\right)^{-\gamma_{n}} \leftarrow G_{n}\left(\mathbb{C}^{\infty}\right)^{-2 \gamma_{n}} \leftarrow \cdots
$$

as a cofinal system, where the maps are induced from $-(N+1) \gamma_{n}+\gamma_{n}=-N \gamma_{n} \in$ $K\left(G_{n}\left(\mathbb{C}^{\infty}\right)\right)$ for all $N \in \mathbb{Z}_{+}$.

Proof. Let $p_{j}=p_{(j, j+1, \ldots, j+n-1)}$ for all $j \in \mathbb{Z}$. Then for $j<-n$ we have $\left.B \mathcal{C}_{f_{A}}\right|_{p_{j}} ^{p_{0}} \cong$ $G_{n}\left(\mathbb{C}\left[z, z^{-1}\right]^{\{j, j+1, \ldots, n-1\}}\right)$. Moreover, the normal bundle of $\left.\left.B \mathcal{C}_{f_{A}}\right|_{p_{j}} ^{p_{0}} \hookrightarrow B \mathcal{C}_{f_{A}}\right|_{p_{j}} ^{p_{m}}$ is $m \gamma_{n}$ for all $m \in \mathbb{N}$. Hence $\left\{\left(\left.B \mathcal{C}_{f_{A}}\right|_{p_{j}} ^{p_{0}}\right)^{-\nu_{a}}\right\}_{a \in I_{p_{j}}}$ has

$$
G_{n}\left(\mathbb{C}\left[z, z^{-1}\right]^{\{j, j+1, \ldots, n-1\}}\right) \leftarrow G_{n}\left(\mathbb{C}\left[z, z^{-1}\right]^{\{j, j+1, \ldots, n-1\}}\right)^{-\gamma_{n}} \leftarrow \cdots
$$

as a cofinal system. Taking a direct limit as $j \rightarrow-\infty$, we see that

$$
G_{n}\left(\mathbb{C}\left[z, z^{-1}\right]^{\{\ldots n-2, n-1\}}\right) \leftarrow G_{n}\left(\mathbb{C}\left[z, z^{-1}\right]^{\{\ldots n-2, n-1\}}\right)^{-\gamma_{n}} \leftarrow \cdots
$$

is a cofinal system of the Floer homotopy type of $f_{A}: G_{n}\left(\mathbb{C}\left[z, z^{-1}\right]\right) \rightarrow \mathbb{R}$.

Note that the Floer cohomology module computed algebraically in the previous section can be recovered from the above cofinal system using the Thom Isomorphism Theorem as the direct limit of the following:

$$
H^{*}\left(G_{n}\left(\mathbb{C}^{\infty}\right)\right) \stackrel{\cup c_{n}}{\longrightarrow} H^{*+2 n}\left(G_{n}\left(\mathbb{C}^{\infty}\right)\right) \stackrel{\cup c_{n}}{\longrightarrow} H^{*+4 n}\left(G_{n}\left(\mathbb{C}^{\infty}\right)\right) \stackrel{\cup c_{n}}{\longrightarrow} \cdots
$$

Hence the Floer cohomology $f_{A}: G_{n}\left(\mathbb{C}\left[z, z^{-1}\right]\right) \rightarrow \mathbb{R}$ is recoverable from its Floer homotopy type.

\section{REFERENCES}

1. M. Audin and J. Lafontaine Editors. Holomorphic Curves in Symplectic Geometry. Birkhäuser Verlag, Basel, 1994. MR 95i:58005

2. D.M. Austin and P.J. Braam. Morse-Bott theory and equivariant cohomology. In H. Hofer et. al., editors, The Floer Memorial Volume, Birkhäuser, Basel, 1995, pages 123-183. MR 96i: 57037

3. S. Berceanu and A. Gheorghe. On the construction of perfect Morse functions on compact manifolds of coherent states. J. Math. Phys., 28(12):2899-2907, 1987. MR 88m:58026

4. R. Bott. An application of Morse theory to the topology of Lie groups. Bull. Soc. Math. France, 84:251-281, 1956. MR 19:291a 
5. T. Bröcker and T. tom Dieck. Representations of Compact Lie Groups. Springer-Verlag, New York, 1985. MR 86i:22023

6. R.L. Cohen. Morse theory via moduli spaces. Mat. Contemp., 2:19-66, 1992. MR 95m:58026

7. R.L. Cohen, J.D.S. Jones, and G.B. Segal. Morse theory and classifying spaces. Preprint, 1992.

8. R.L. Cohen, J.D.S. Jones, and G.B. Segal. Floer's infinite dimensional Morse theory and homotopy theory. In H. Hofer et. al., editors, The Floer Memorial Volume, Birkhäuser, Basel, 1995, pages 297-325. MR 96i:55012

9. A. Floer. An instanton-invariant for 3-manifolds. Comm. Math. Phys., 118:215-240, 1988. MR 89k: 57028

10. A. Floer. Morse theory for Lagrangian intersections. J. Diff. Geom., 28:513-547, 1988. MR 90f: 58058

11. A. Floer. Symplectic fixed points and holomorphic spheres. Comm. Math. Phys., 120:575-611, 1989. MR 90e: 58047

12. T. Frankel. Fixed points and torsion on Kahler manifolds. Ann. of Math., 70(1):1-8, 1959. MR 24:A1730

13. D. Hurtubise. The Floer homotopy type of complex Grassmann manifolds. Preprint, 1996.

14. J. Milnor. Morse Theory. Princeton University Press, Princeton, New Jersey, 1969. MR 29:634

15. J. Milnor and J. Stasheff. Characteristic Classes. Princeton University Press, Princeton, New Jersey, 1974. MR 55:13428

16. M. Schwarz. Morse Homology. Birkhäuser, Boston, 1993. MR 95a:58022

17. R. Stanley. Some combinatorial aspects of the Schubert calculus. In P.A. Meyer, editor, Combinatoire et Représentation du Groupe Symétrique, Lecture Notes in Math. vol. 579, pages 217-251, 1977. MR 57:5766

18. F. Warner. Foundations of Differentiable Manifolds and Lie Groups. Springer-Verlag, New York, 1983. MR 84k:58001

Department of Mathematics, Occidental College, 1600 Campus Drive, Los Angeles, California 90041

E-mail address: hurtubis@oxy.edu 